\title{
A Study of the Multisensor Estimation Method Based on Fusion Technology for Subsurface Defect Depth
}

\author{
Ren Tiao-juan, ${ }^{1}$ Liu Ban-teng $\mathbb{D},{ }^{1}$ Chen You-rong $\mathbb{D},{ }^{1}$ Yang Haibo $\mathbb{D}^{1},{ }^{1}$ and Zhu Yun-kai $\mathbb{D}^{2}$ \\ ${ }^{1}$ College of Information Engineering, Zhejiang Shuren University, Hangzhou, Zhejiang, China \\ ${ }^{2}$ Zhejiang Hangjia Technology Development Co. Ltd., Hangzhou, China
}

Correspondence should be addressed to Liu Ban-teng; hupo3@sina.com

Received 13 September 2017; Revised 5 February 2018; Accepted 12 February 2018; Published 11 April 2018

Academic Editor: Luca Francioso

Copyright (C) 2018 Ren Tiao-juan et al. This is an open access article distributed under the Creative Commons Attribution License, which permits unrestricted use, distribution, and reproduction in any medium, provided the original work is properly cited.

\begin{abstract}
In consideration of difficulty in directly using the multisensor detecting feature information for the defect identification, an improved multisensor recognition algorithm based on data-fusion technology for subsurface defect depth evaluation is proposed. At first, two common nondestructive testing technologies such as ultrasonic testing (UT) and eddy current testing (ECT) are introduced; then, a fusion method based on the error distribution characteristics of two kinds of detection methods is improved through the hyperbolic discriminant function to evaluate the subsurface defect depth. The experimental result shows that the improved algorithm is superior to the existing algorithm, so it can achieve better synthesis results and improve the correct recognition rate.
\end{abstract}

\section{Introduction}

The quantified nondestructive testing for the subsurface defect of conductive parts in large components, such as fatigue crack and stress corrosion in fastening structure or lap joints, has been a pressing research subject in key fields like aircraft, aviation, rail transit, and processing and manufacturing industry [1]. As an important part in detection technology, nondestructive testing is a comprehensive process of testing, crack detection, evaluation, and so on, without damage to own physical or structural characteristics of detect objects $[2,3]$, and it plays a key role in controlling and improving product quality and ensuring the reliability of the material, component, products, and equipment, which is an inseparable part in modern industrial production and has become a hot field to be studied by professional scholars home and abroad [4-7]. The method using magnetooptic imaging sensor to detect the subsurface defect shape by combining the eddy current effect and Faraday photoelectric effect is proposed in [4], which takes an advantage in highspeed, real-time, large-area, and visualization. The quantified testing method based on water immersed ultrasonic sensor is studied in [5], which validates the defect echo in both concave and convex surface. The method solves the high-cost and low applicability problem which happens in the traditional method. A simple procedure, strong-practicality, and high-efficiency nondestructive method is proposed in [6], which uses the penetration method to test the weld defect in the high-speed bullet train. The method using the eddy current sensor to test the subsurface defect is studied in [7], which validates its efficiency with the finite element method.

Because of the respective feature and limited applicable scope of the single sensor, which cannot overall detect various subsurface defects, multisensor-integrated nondestructive testing techniques are concerned and studied more and more. It mainly involves signal processing, numerical modelling, data fusion, inverse analysis, and other fields, which belongs to the research area of advanced detection technique $[8,9]$. The basic study and application of multisensorintegrated nondestructive testing theory and technique have become an important research trend in many countries such as America, Britain, and Russia. Domestically, with the manufacture of key aviation aircraft, mass production stage of aerial product and the booming development of rail transit, 
nuclear power plants, and rapid and reliable testing requirements for the integrated nondestructive testing is becoming increasingly urgent.

As to the puzzle that various feature information from different sensors cannot directly use on the depth testing of the subsurface defect, an algorithm based on multisensor fusion technology used for the subsurface defect depth estimation is proposed. Multisensor information fusion technique originated from the 1970 s and is wisely applied to various intelligent platforms and civil fields. Multisensor information fusion technique is actually a functional analogy in dealing the complicated problem of the human brain. Compared with the single sensor, the multisensor information fusion technique can enhance system survival ability and improve the reliability and robustness of the whole system when solving the problem of detection, tracking, and target recognition. As one of the research hotspot in multisensor detection, experts both home and abroad have carried out extensive research and promote many information fusion techniques [10, 11]. At present, different algorithms of date fusion are researched, which contains weighted average fusion, Kalman filtering method, Bayes estimation, statistical decision theory, probability method, fuzzy logic inference, artificial neural network, D-S evidence theory, and so on. A weighted average fusion method used in calculating the proportion of the effective pixels from each data source is proposed in [10], which reduces the source data noise and improves data space coverage and confidence. The multisensor Kalman filtering fusion method is proposed in [11], which has high filtering precision and can eliminate measurement system error successfully. A multisensor fusion algorithm of electronic circuit fault orientation based on D$S$ evidence theory is proposed in [12], which shows the superiority of multisensor fusion computing method. A transformer fault diagnosis method based on multiple neural network and evidence theory fusion is proposed in [13], which organic combines the neural network and evidence theory for the data fusion.

ECT and UT are the typical nondestructive method, but both of them have advantages and disadvantages. When the depth of the defect is on the shallow surface, the reliability of eddy current testing is higher than ultrasonic testing and when the depth of defect is in the inner deep, the ultrasonic testing is more reliable. In order to overcome limitations of the single detection method for defect on the subsurface, a quantitative detection algorithm based on data fusion is proposed in this paper.

\section{The Quantitative Detection Model of ECT and $U T$}

The respective detection mathematical model of ECT and UT is built through machine learning of massive testing data firstly. ECT theory is derived from Maxwell's equations, which is a nondestructive testing method based on the principle that the eddy current will generate when the conductor is in the alternating magnetic field. The eddy current in the conductors reacts to the detecting magnetic coil and produces impedance in the magnetic coil correspondingly, which contributes to the calculation of amplitude, phase, and flow form of eddy current. Thus, according to these information, the specific defect status of the test specimen can be obtained by comparing with the standard objects.

Through analysing the ECT theory, there are some constraints on using eddy current testing method due to the conductor skin effect in changing electromagnetic field, eddy current density attenuates rapidly with an increase of the defect depth. The attenuation speed is described as

$$
I_{x}=I_{0} e^{-\sqrt{\pi f \mu \sigma} x} .
$$

In (1), $I_{0}$ is the eddy current density on the infinite conductor surface, $f$ is the excitation frequency of alternating current, $\mu$ is the magnetic permeability of the materials, and $\sigma$ is the electrical conductivity of the materials. $I_{x}$ is the eddy current density in the depth $x$ from the surface. As this equation shows the reliability of eddy current testing, it will be less and less with the depth increasing. The standard surface effect depth is defined as the depth when the eddy current density attenuates to $1 / e$ from the surface as

$$
\delta=\frac{1}{\sqrt{\pi f \mu \sigma}} .
$$

In general, the material of the testing object is stationary; thus, the electrical conductivity $\sigma$ and magnetic permeability $\mu$ is almost constant. The depth of $3 \delta$ from the surface is defined as the limit position of eddy current testing. Based on the knowledge, the spectrum amplitude and the depth of the subsurface defects are in an exponential relationship as

$$
x=\alpha_{1} d^{\beta_{1}}+\gamma_{1} .
$$

$x$ is the amplitude feature got from the eddy current sensor, $d$ is the depth of subsurface defects, $\beta_{1}$ is the depth of defect, and $\alpha_{1}$ and $\gamma_{1}$ are the equilibrium parameters in uncertain conditions. $\beta_{1}$ and $\gamma$ can be calculated by the least square method with massive testing data as

$$
\begin{aligned}
\min F\left(\beta_{1}, \gamma_{1}\right)= & \sum_{i}\left\|x_{i}-\alpha_{1} d_{i}^{\beta_{1}}-\gamma_{1}\right\|^{2} \\
& \Rightarrow\left\{\begin{array}{l}
\frac{\partial F\left(\alpha_{1}, \beta_{1}, \gamma_{1}\right)}{\partial \alpha_{1}}=0 \\
\frac{\partial F\left(\alpha_{1}, \beta_{1}, \gamma_{1}\right)}{\partial \beta_{1}}=0 \\
\frac{\partial F\left(\alpha_{1}, \beta_{1}, \gamma_{1}\right)}{\partial \gamma_{1}}=0
\end{array}\right.
\end{aligned}
$$

In (4), $x_{i}$ is the amplitude feature tested by eddy current testing when the depth of defect is $d_{i} . \alpha_{1}, \beta_{1}$, and $\gamma_{1}$ can be calculated by massive testing data $\left(x_{i}, d_{i}\right)$. All the testing data is from the experiment, and the testing specimens and numbers is explained in Section 4.

The physical foundation of UT is the mechanical vibration of the particle and its propagation, and it follows the law of reflection, refraction, wave superposition, interference, 
Huygens principle, and so on. The pulse reflection ultrasonic detection method is the most widely used which launches ultrasonic wave to the testing material and the echo single is collected to analyze the physical properties of the material by building the corresponding relationship between the reflected sound wave amplitude and the propagation time [14]. The propagation time can be precisely positioned by the horizontal ordinate (time baseline), and the vertical ordinate data can just be used to get the relative values accounting for the shape and distribution of the defects, which can be available for the knowledge of unknown defects by comparing with the standard defects. If there is a defect in the material, the corresponding pulse waveform will appear in the time baseline. As the proportional relationship between horizontal scale and testing depth, the defect depth $d$ is

$$
d=\tau_{f} \frac{T}{\tau_{b}} .
$$

In (5), $\tau_{f}$ is the horizontal scale, $T$ is the reflection time, and $\tau_{b}$ is the time interval. As to the influence of the propagation time which is unable to get defects depth by the existing data, the testing reliable depth can only be evaluated by the data analysis. Based on the datum at hand, the feature data from the subsurface defect testing and the subsurface depth are related to each other by

$$
y=\alpha_{2} d+\beta_{2} .
$$

In (6), $y$ is the echo arrival time feature of ultrasonic testing, $d$ is the depth of subsurface defect, and $\alpha_{2}, \beta_{2}$ are the ultrasonic testing parameters. $\alpha_{2}, \beta_{2}$ can be calculated by the least square method with massive testing data. Its least square method can be described by

$$
\min F\left(\alpha_{2}, \beta_{2}\right)=\sum_{i}\left\|y_{i}-\alpha_{2} d_{i}-\beta_{2}\right\|^{2} \Rightarrow\left\{\begin{array}{l}
\frac{\partial F\left(\alpha_{2}, \beta_{2}\right)}{\partial \alpha_{2}}=0, \\
\frac{\partial F\left(\alpha_{1}, \beta_{2}\right)}{\partial \beta_{2}}=0 .
\end{array}\right.
$$

In (7), $y_{i}$ is the echo arrival time feature tested by ultrasonic testing when the depth of defect is $d_{i}$. $\alpha_{1}, \beta_{2}$ can be calculated by massive testing data $\left(y_{i}, d_{i}\right)$.

Through both the ECT and UT method, the feature data can be got by a massive subsurface material with known depth, and the subsurface defect depth can be evaluated, and then the reliability and error distribution can be obtained. The reliability of two methods can be described by RMSE (root mean square error) as

$$
\begin{aligned}
& R_{x}=\sqrt{\frac{\sum_{i}\left\|x_{i}-\alpha d_{i}^{\beta_{1}}-\gamma_{1}\right\|^{2}}{n}}, \\
& R_{y}=\sqrt{\frac{\sum_{i}\left\|y_{i}-\alpha_{2} d_{i}-\beta_{2}\right\|^{2}}{n}} .
\end{aligned}
$$

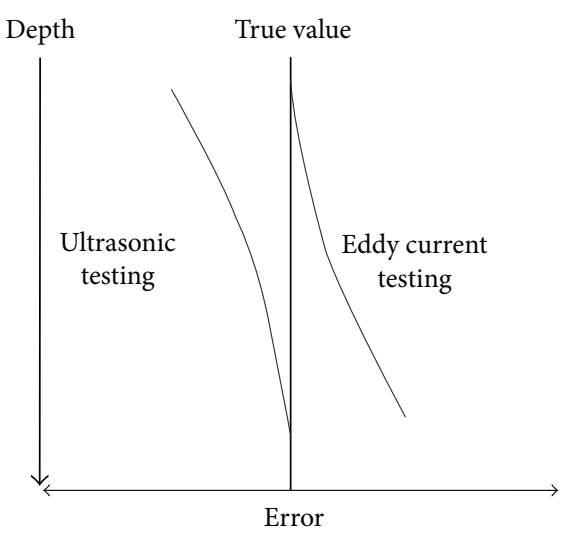

FIgURE 1: Error distribution.

In (8), $R_{x}$ is the unreliability of eddy current testing, and $R_{y}$ is the unreliability of ultrasonic testing which can be described in Figure 1 by massive testing data.

Both of the two nondestructive testing methods have advantages and disadvantages as shown in Figure 2. The ultrasonic testing has advantages of convenient operation, accurate positioning, and high sensitivity when using the defect echo time and amplitude to value the defect depth, but it takes the disadvantages of the poor testing ability to the surface and near-surface defects. In the detection progress, the defect information will be hidden in the first echo when the defect depth is small; according to the detection error, ultrasonic quantitative estimation error is small (less than $0.5 \mathrm{~mm}$ ) if the defect depth is deeper than $3.6 \mathrm{~mm}$. When the depth of the defects is shallow, the eddy current testing is more reliable than the ultrasonic testing; according to the detection error, eddy current quantitative estimation error is small (less than $0.5 \mathrm{~mm}$ ) if the defect depth is shallower than $2.8 \mathrm{~mm}$, while in the deep depth, the ultrasonic testing is more reliable than the eddy current testing.

\section{Defect Depth Quantitative Evaluation Algorithm of Multisensors}

For error distribution characteristics of the two detection methods, the subsurface defect quantitative assessment algorithm is proposed, which is based on the multisensor data theory.

In order to eliminate the different dimensions between the ECT and UT, the testing data have to be normalized. In this paper, the min-max method is used for processing data, and the processed data meet the requirements of the standard normal distribution (the mean is 0 and the standard deviation is 1). Where $\chi^{*}$ is the dimensionless characteristic value. $\chi$ is the observational data of the eddy current sensor or ultrasonic sensor. $\chi_{\min }$ is the minimum value of each group, and $\chi_{\max }$ is the maximum value of each group.

$$
\chi^{*}=\frac{\chi-\chi_{\min }}{\chi_{\max }-\chi_{\min }} .
$$

After acquirement of the dimensionless characteristic value of the two kinds of measurement methods, the defect 


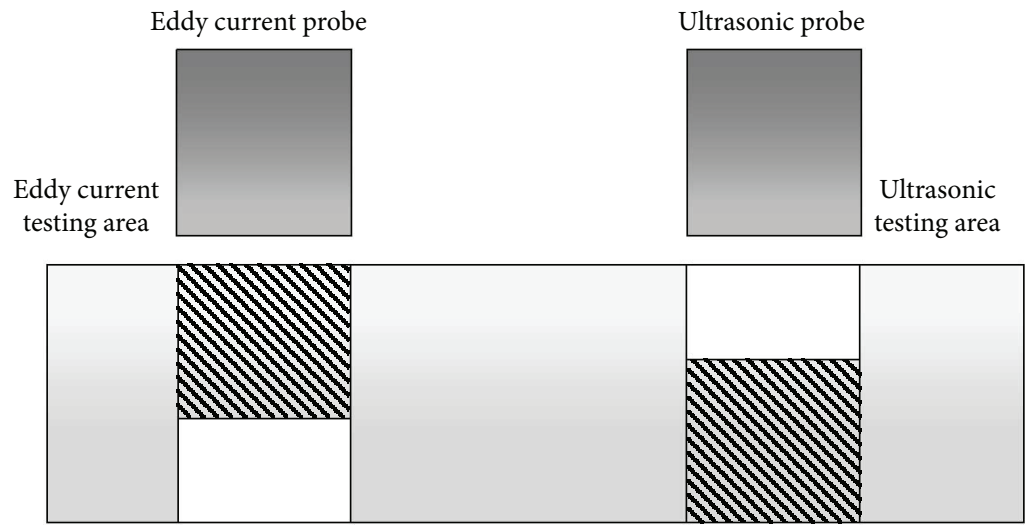

FIGURE 2: Integrated detection schematic.

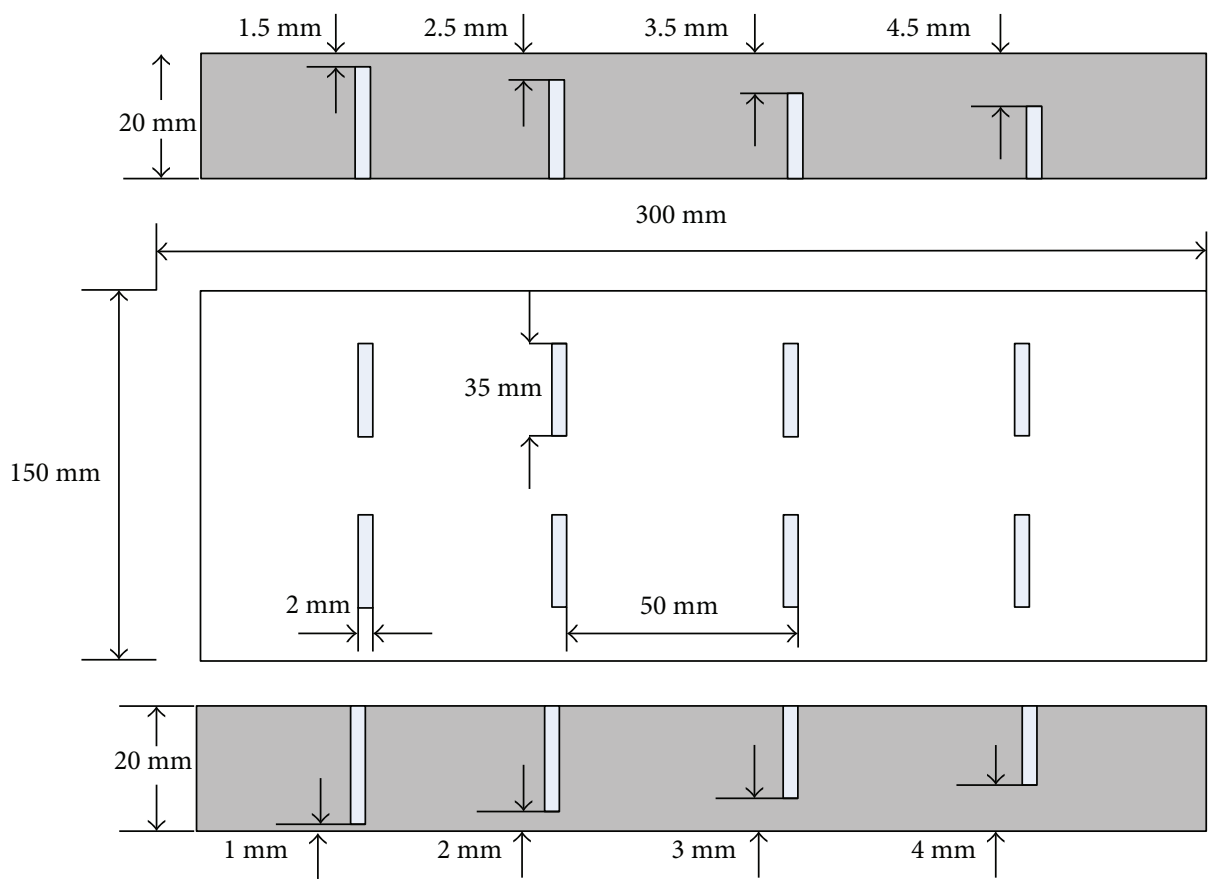

FIgure 3: The schematic diagram of the specimen and defects.

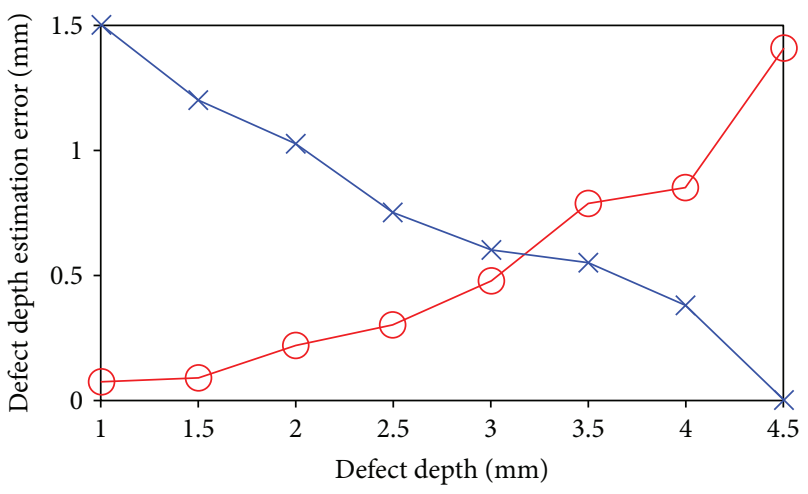

ECT

$\leftarrow$ UT

FIGURE 4: The error change curve.

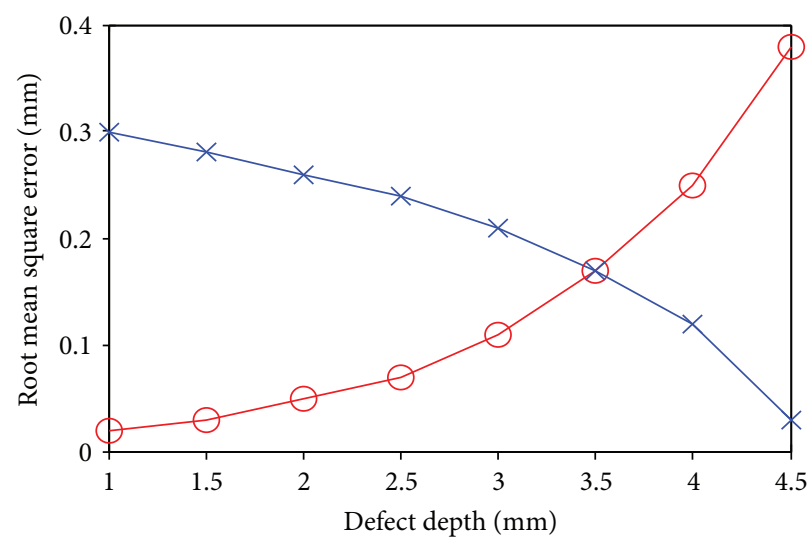

$\bigcirc$ ECT

$\star$ UT

Figure 5: The unreliability change curve. 
TABLE 1: The evaluation results of the surface material defect depth using three methods.

\begin{tabular}{lcccccc}
\hline Number & $\begin{array}{c}\text { The real } \\
\text { defect depth }\end{array}$ & $\begin{array}{c}\text { Echo arrival time feature (ms) of } \\
\text { ultrasonic testing }\end{array}$ & $\begin{array}{c}\text { Amplitude feature (V) of } \\
\text { eddy current testing }\end{array}$ & $\begin{array}{c}\text { The depth } \\
\text { of UT }\end{array}$ & $\begin{array}{c}\text { The depth } \\
\text { of ECT }\end{array}$ & $\begin{array}{c}\text { The depth of } \\
\text { multisensor testing }\end{array}$ \\
\hline 1 & 4.5 & 21.48 & 0.578 & 4.49 & 3.05 & 4.49 \\
2 & 4 & 21.09 & 0.574 & 4.36 & 3.13 & 4.36 \\
3 & 3.5 & 20.56 & 0.583 & 4.13 & 2.72 & 3.75 \\
4 & 3 & 19.88 & 0.587 & 3.69 & 2.49 & 3.17 \\
5 & 2.5 & 19.21 & 0.593 & 3.27 & 2.22 & 1.81 \\
6 & 2 & 18.43 & 0.601 & 2.99 & 2.64 & 1.44 \\
7 & 1.5 & 18.27 & 0.607 & 2.53 & 1.04 & 1.44 \\
8 & 1 & 18.08 & 0.612 & & 1.04 \\
\hline
\end{tabular}

depth prediction methods of the ECT and UT can be used, respectively, namely, $d^{s}$ and $d^{e}$. Where $d^{s}$ is the predicted depth of eddy current testing from the (3). And the $d^{e}$ is the predicted depth of ultrasonic testing from the (6).

Usually, two kinds of detection methods do not take effect at the same time, $d^{s} \neq d^{e}$. And even then, there is the huge difference between the results of these two methods. At this point, it is necessary to construct the model for evaluating which kind of detection method is more reliable. A hyperbolic discriminate function model is proposed

$$
j\left(d^{s}, d^{e}\right)=\frac{1-e^{-\theta_{1}\left(d^{s}-\theta_{3}\right)}}{1+e^{-\theta_{1}\left(d^{s}-\theta_{3}\right)}}-\frac{1-e^{-\theta_{2}\left(d^{e}-\theta_{3}\right)}}{1+e^{-\theta_{2}\left(d^{e}-\theta_{3}\right)}},
$$

where $d^{s}$ and $d^{e}$ are the surface defect depths got from the two kinds of detection methods. $\theta_{1}, \theta_{2}$, and $\theta_{3}$ are the evaluation parameters of the integrated detection method, which can be got from the least square calculation based on massive testing data.

The defect depth can be obtained through this discriminate model based on the decision-making mode by maximizing the accurate determination of probability, where $d$ is the depth which is got from the eddy current or ultrasonic testing model. Through several iterations, the depths corresponding to different interval value are given in the following situations.

Discriminate model 1: when $d$ tends to the deep location (deeper than $3.6 \mathrm{~mm}$ ), the first item of $(10)\left(\left(1-e^{-\theta_{1}\left(d^{s}-\theta_{3}\right)}\right) /\right.$ $\left.\left(1+e^{-\theta_{1}\left(d^{s}-\theta_{3}\right)}\right)\right)$ tends to be 1 , while the latter item usually tends to be 0 . According to this model similar to the step function, the conclusion can be obtained: ultrasonic testing result is effective, and eddy current testing result is invalid. Formula (5) is use to calculate the defect depth.

Discriminate model 2: when $d$ tends to the location of the shallow (shallower than $2.8 \mathrm{~mm}$ ), the second item of (10) $\left(\left(1-e^{-\theta_{2}\left(d^{e}-\theta_{3}\right)}\right) /\left(1+e^{-\theta_{2}\left(d^{e}-\theta_{3}\right)}\right)\right)$ tends to be 1 , while the former item usually tends to be 0 . Based on this property that $j\left(d^{s}, d^{e}\right) \rightarrow-1$, the conclusion can be obtained: eddy current testing results are effective, and ultrasonic testing result is invalid. Equation (3) is used to calculate the defect depth.

Discriminate model 3: when $d$ does not have special trend in depth (between the $2.8 \mathrm{~mm}$ and $3.6 \mathrm{~mm}$ ), the absolute value of $j\left(d^{s}, d^{e}\right)$ is not too big either. In such case, the single model of the eddy current testing or ultrasonic testing is no longer used, but the equilibrium model of uncertainty will be used to calculate the defect depth as

$$
G\left(d^{s}, d^{e}\right)=\frac{1-e^{-\gamma_{1}\left(d_{i}^{s}-\gamma_{3}\right)}}{1+e^{-\gamma_{1}\left(d_{i}^{s}-\gamma_{3}\right)}} x+\frac{1-e^{-\gamma_{2}\left(d_{k}^{e}-\gamma_{3}\right)}}{1+e^{-\gamma_{2}\left(d_{k}^{e}-\gamma_{3}\right)}} y
$$

$x$ and $y$ are the characteristics of the data obtained under two kinds of detecting methods, respectively. $\gamma_{1}, \gamma_{2}$, and $\gamma_{3}$ are the parameters of the data fusion, which can be achieved by the least square method, respectively.

\section{The Simulation Analysis}

In order to validate the performance of the proposed multisensor recognition algorithm based on fusion technology for subsurface defect depth, the ultrasonic testing and eddy current testing are used to obtain the feature data. The experimental samples contain 400 groups of materials (aluminum) which have the defect depth of $1 \mathrm{~mm}, 1.5 \mathrm{~mm}, 2 \mathrm{~mm}, 2.5 \mathrm{~mm}$, $3 \mathrm{~mm}, 3.5 \mathrm{~mm}, 4 \mathrm{~mm}$, and $4.5 \mathrm{~mm}$ and each defect is tested 50 times. 320 groups of data are used to calculate the relative parameters, statistical error, and the statistical unreliability of these two detection methods, and 80 groups of data are used to verify the accuracy of the testing method. The schematic diagram of the specimen and defects is shown in Figure 3. The statistical error and the statistical unreliability curve changed with the depth of the defect are shown in Figures 4 and 5. The result of the 80 groups of data's average quantitative assessments is presented in Table 1.

The results of Table 1 are shown in Figures 6-8. According to the figures, the absolute error and relative error of the method proposed in this paper perform better than the other methods. In the detection of the shallow defect depths, the absolute error and relative error of UT are large. In the detection of the deep defect depths, the relative error of the ECT is large. Comparing with the unreliability (the root mean square) of the three detecting methods, the method proposed in this paper is the most reliable method in Figure 8.

\section{Conclusion}

In order to overcome the limitation of the depth detection method through the single sensor, an improved multisensor recognition algorithm based on data fusion technology for 


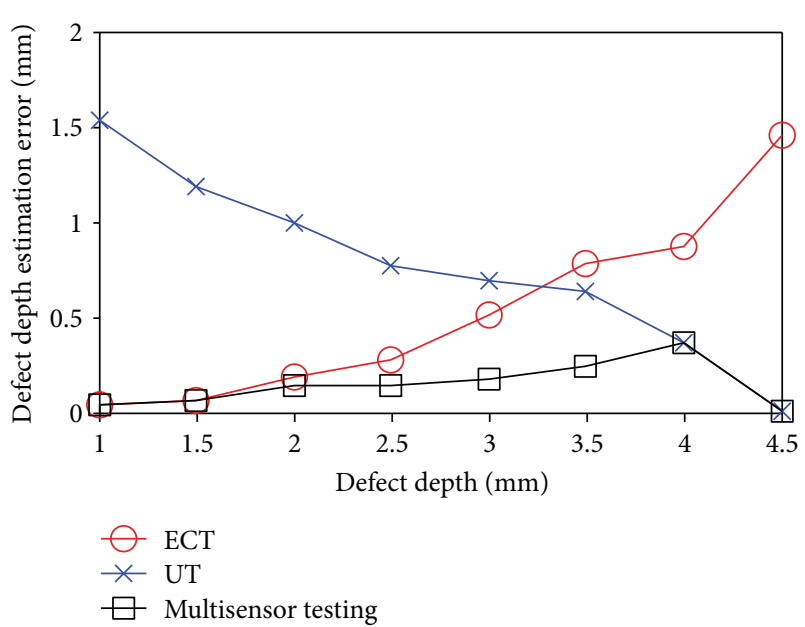

FIgURE 6: The absolute error distribution of the defect depths.

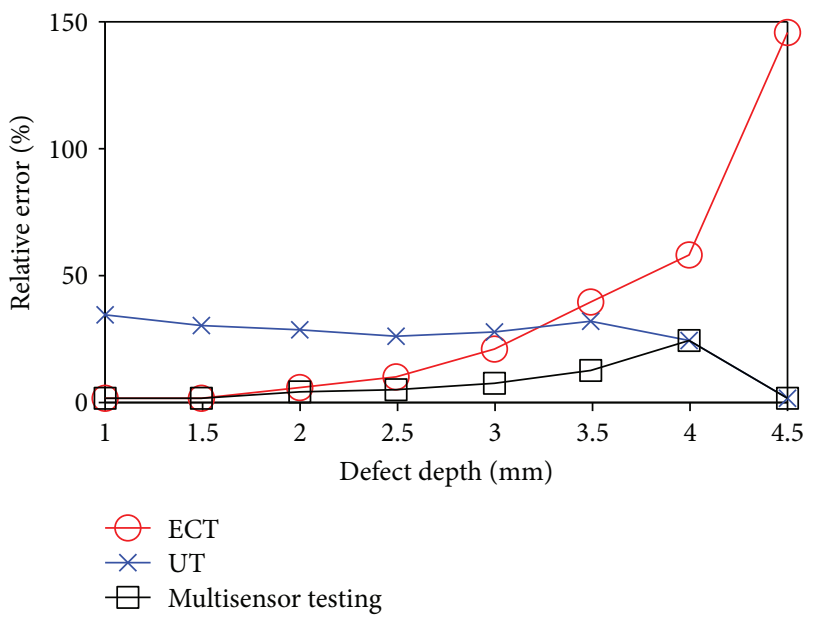

FIgURE 7: The relative error distribution of the defect depths.

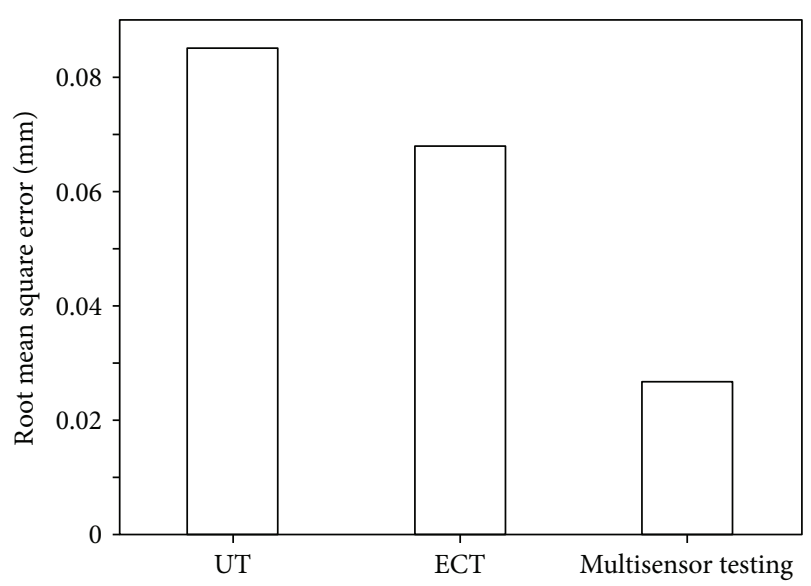

Figure 8: The root mean square error distribution of the defect.

subsurface defect depth evaluation is proposed in this paper. The kernel idea of this algorithm is to build the discriminate function and fusion function of ECT and UT data. The discriminate function is used to evaluate all kinds of reliability test method and when the discriminate function fails to effectively distinguish the defects, the fusion function will be used to make the quantitative assessment of the depth of the surface defects with the multiple sensor data. The result of the simulation experiment shows that this algorithm has a higher accuracy rate.

\section{Conflicts of Interest}

The authors declare that they have no conflicts of interest.

\section{Acknowledgments}

The authors acknowledge the support of the Zhejiang provincial Public Welfare Technology Application and Research Projects of China (LGF18F010005, 2016C33038), Zhejiang Provincial Major Science and Technology Special Project of China (2015C01033), and National Natural Science Foundation of China (61501403).

\section{References}

[1] Q. Wang, Q. Bai, J. Chen, Y. Sun, Y. Guo, and Y. Liang, "Subsurface defects structural evolution in nano-cutting of single crystal copper," Applied Surface Science, vol. 344, pp. 38-46, 2015.

[2] V. Arjun, B. Sasi, B. Purna Chandra Rao, C. K. Mukhopadhyay, and T. Jayakumar, "Optimisation of pulsed eddy current probe for detection of sub-surface defects in stainless steel plates," Sensors and Actuators A: Physical, vol. 226, pp. 6975, 2015.

[3] S. Grys, L. Vokorokos, and L. Borowik, "Size determination of subsurface defect by active thermography - simulation research," Infrared Physics \& Technology, vol. 62, pp. 147153, 2014.

[4] X. Xin, Research on Ferromagnetic Materials Surface Defects Detection Using Magneto-Optic Imaging Method, University of Electronic Science and Technology of China, Chengdu, 2014.

[5] H. W. Hu, L. X. Peng, Z. G. Zhou et al., "Quantitative research on defect of curved components with immersion ultrasonic testing," Acta Aeronautica et Astronautica Sinica, vol. 35, no. 11, pp. 3166-3173, 2014.

[6] C. Hongyu, Y. Yu, J. Gao et al., "Application of penetrant test to $\mathrm{Al}$ alloy train body," Welding \& Joining, vol. 1, pp. 61-63, 2015.

[7] L. Yansong, Studies of Frequency-Domain Feature Extraction and Defect Characterization in Pulsed Eddy Current Testing, Xiamen University, Xiamen, 2014.

[8] S. Xue-hui, L. Xiao-bing, Z. Yan, and Q. Peng-peng, "Multisensor fusion target tracking of reentry phase based on square-root unscented Kalman filter," Systems engineering and Electronics, vol. 34, no. 2, pp. 303-306, 2012.

[9] G. Zhong-Wen, W. Xi, H. Nai-Jun, and Q. Li-Ke, "An event detection algorithm based on multi-sensor data fusion," Periodical of Ocean University of China, vol. 44, no. 10, pp. 155160, 2014.

[10] C. Yunzhi, X. Wang, B. Wu, and S. Liya, "Ocean color data merging based on adaptive weighted averaging," Remote Sensing Technology and Application, vol. 27, no. 3, pp. 333-338, 2012. 
[11] L. Ma, Z. Man, and C. Jingguang, "Multi-sensor centralized incremental Kalman filtering fusion algorithm," Computer Engineering and Applications, vol. 11, pp. 229-232, 2014.

[12] Z. Da-qi and Y. Sheng-lin, "Data fusion algorithm based on DS evidential theory and its application for circuit fault diagnosis," Acta Electronica Sinica, vol. 30, no. 2, pp. 153-155, 2002.

[13] L. Rui-jin, L. Yu-xiang, Y. Li-jun, and W. You-yuan, "Study on synthetic diagnosis method of transformer fault using multineural network and evidence theory," Proceedings of the CSEE, vol. 26, no. 3, pp. 119-124, 2006.

[14] K. Guo and W. Li, "Combination rule of D-S evidence theory based on the strategy of cross merging between evidences," Expert Systems with Applications, vol. 38, no. 10, pp. 1336013366, 2011. 


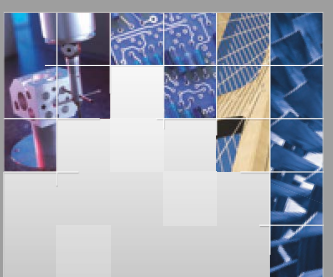

\section{Enfincering}
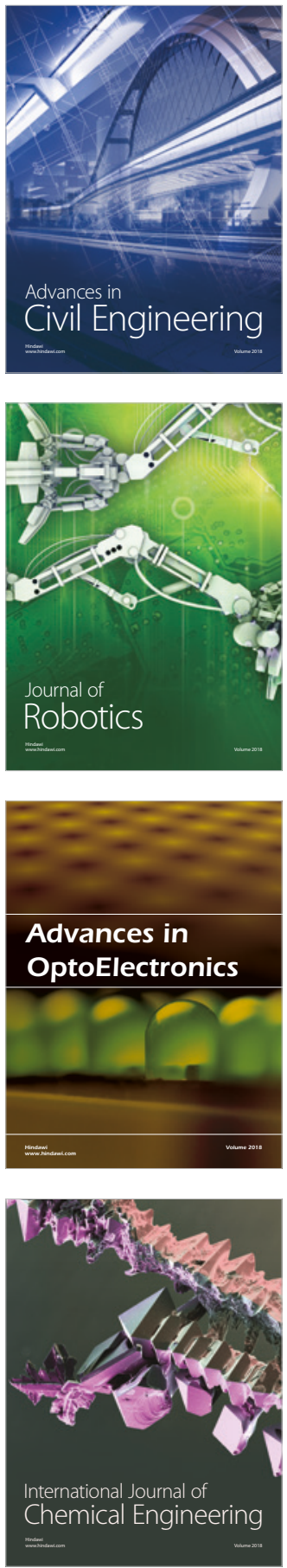

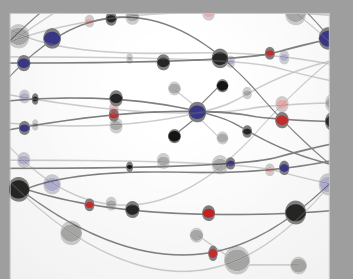

\section{Rotating \\ Machinery}

The Scientific World Journal

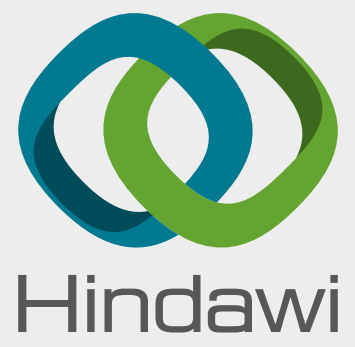

Submit your manuscripts at

www.hindawi.com
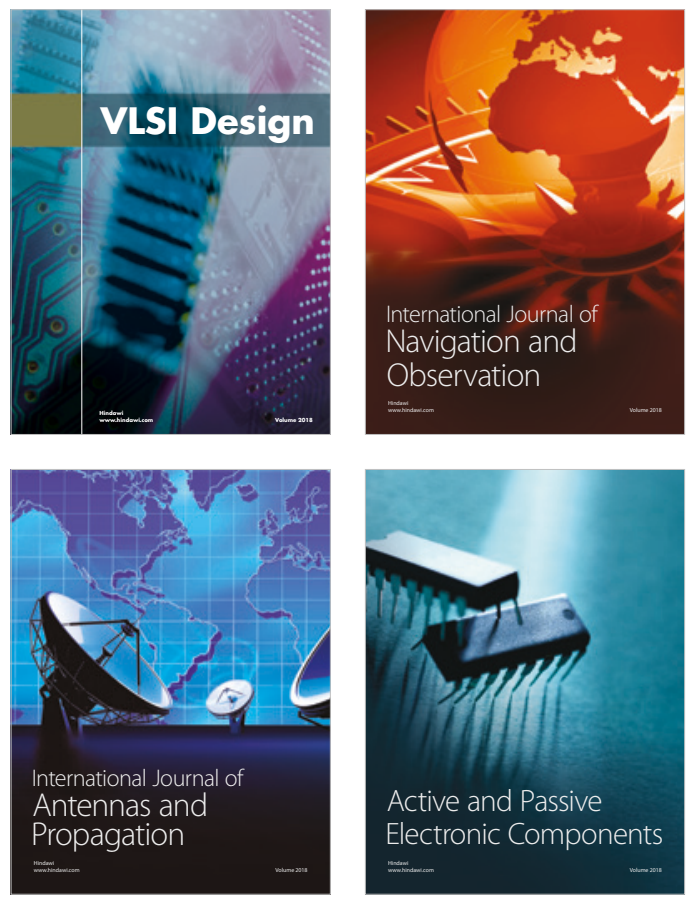
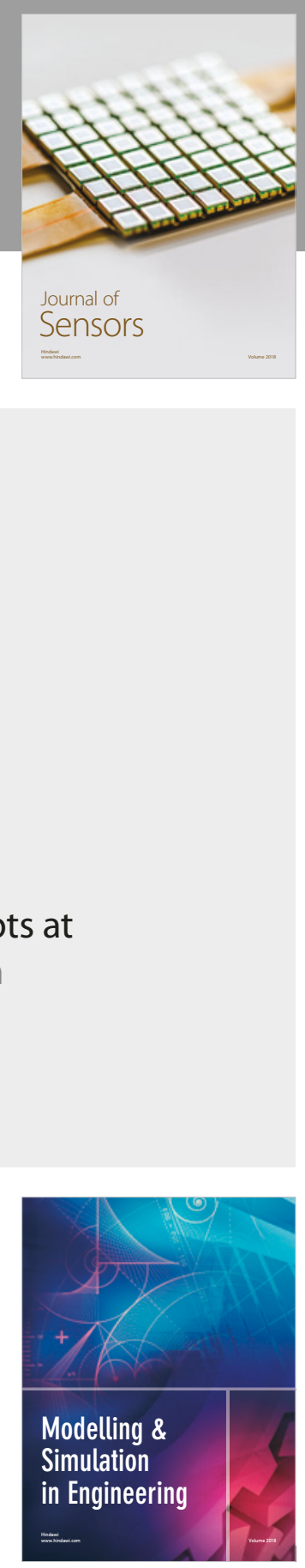

\section{Advances \\ Multimedia}
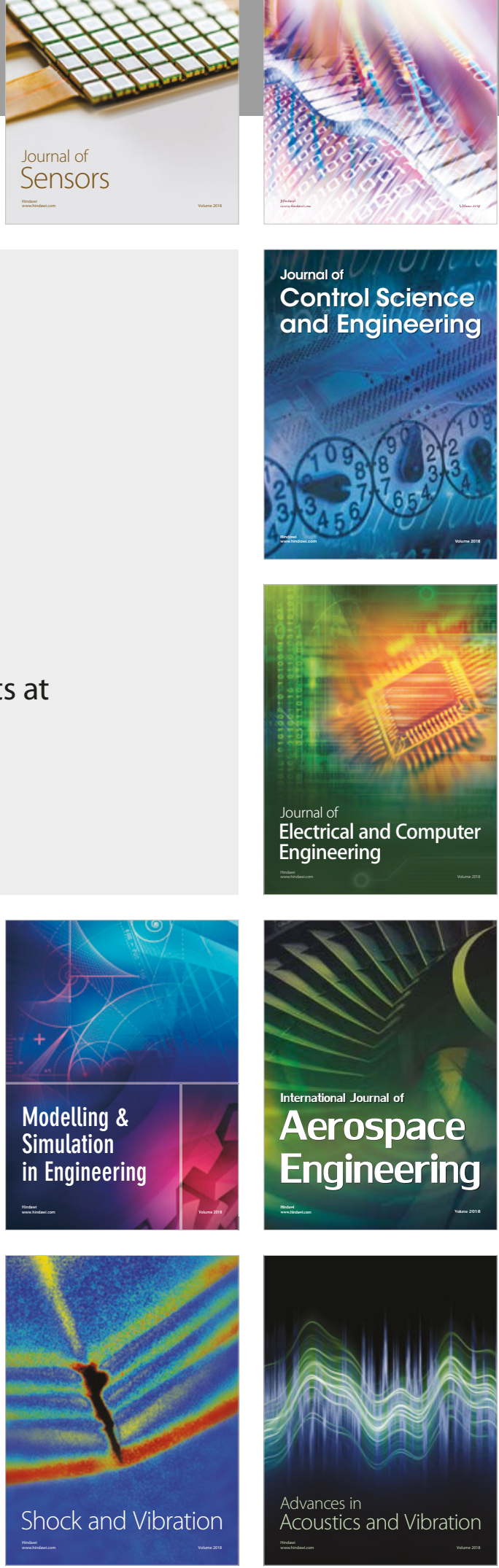